\title{
Higher Serum Testosterone is associated with Increased Risk of Advanced Hepatitis C-related Liver Disease in Males
}

\author{
Donna L. White, $\mathbf{P h D}^{1,2,3}$, Shariar Tavakoli-Tabasi, MD ${ }^{4,5}$, Jill Kuzniarek, BS ${ }^{1,3}$, Rhia \\ Pascua, MPH ${ }^{1,3}$, David J. Ramsey, PhD ${ }^{1,3}$, and Hashem B. El-Serag, MD, MPH ${ }^{1,2,3}$ \\ ${ }^{1}$ Clinical Epidemiology and Outcomes Program, Houston VA Health Services Research and \\ Development Center of Excellence, Michael E. DeBakey Veterans Affairs Medical Center, \\ Houston, Texas \\ 2Section of Gastroenterology and Hepatology, Department of Medicine, Baylor College of \\ Medicine and Michael E. DeBakey Veterans Affairs Medical Center, Houston, Texas \\ ${ }^{3}$ Section of Health Services Research, Department of Medicine, Baylor College of Medicine and \\ Michael E. DeBakey Veterans Affairs Medical Center, Houston, Texas \\ ${ }^{4}$ Hepatitis C Clinic, Section of Infectious Diseases, Michael E. DeBakey Veterans Affairs Medical \\ Center, Houston, Texas \\ ${ }^{5}$ Section of Infectious Diseases, Department of Medicine, Baylor College of Medicine, Houston, \\ Texas
}

\section{Abstract}

Background-Males have strikingly increased risk of advanced liver disease. However, the association between testosterone and risk of hepatitis $\mathrm{C}$ virus (HCV)-related advanced liver disease is unknown.

\begin{abstract}
Methods-We performed a cross-sectional study in male veterans with chronic HCV. Blood samples were obtained to measure total serum testosterone and perform the FibroSURE-ActiTest. Other risk factor data were obtained through systematic questionnaires (e.g., alcohol), physical measurements (e.g., BMI) and serological tests (e.g., viral load). The association between total testosterone and risk of advanced hepatic fibrosis (F3 and F3/F4) and inflammatory activity (A3 and A2/3) measured by FibroSURE-ActiTest was evaluated with logistic regression.
\end{abstract}

\begin{abstract}
Results-A total of 308 eligible study participants were prospectively recruited (mean age 57, 52\% African-American). There were 105 cases with advanced fibrosis and 203 mild fibrosis controls; and 88 cases with advanced inflammatory activity and 220 mild activity controls. Mean total serum testosterone was significantly higher in advanced fibrosis cases as well as advanced inflammatory activity cases compared to mild disease controls $(6.0 \mathrm{ng} / \mathrm{ml} \mathrm{vs.} 5.3 \mathrm{ng} / \mathrm{ml}$ and $5.9 \mathrm{ng} /$ $\mathrm{ml}$ vs. $5.4 \mathrm{ng} / \mathrm{ml}$, respectively). We observed a significant $27 \%$ increase in advanced fibrosis risk and $16 \%$ increase in advanced inflammatory activity risk for each $1 \mathrm{ng} / \mathrm{ml}$ increase in total serum testosterone. Total testosterone in the upper tertile was associated with an even greater excess risk of advanced fibrosis than advanced inflammatory activity $\left(\mathrm{OR}_{\text {adjusted advanced fibrosis }}=3.78,95 \% \mathrm{CI}\right.$ $1.88-7.61$ vs. $\mathrm{OR}_{\text {adjusted advanced inflammatory activity }}=2.64,95 \%$ CI $1.29-5.45$, respectively).
\end{abstract}

Correspondence author: Donna L. White, PhD, Michael E. DeBakey VA Medical Center, 2002 Holcombe Blvd. (MS 152), Houston, Texas 77030, dwhite1@bcm.edu.

The authors declare no conflict of interest. The U.S. Department of Veteran Affairs, the National Institutes of Health, and the National Institute of Diabetes Digestive and Kidney Disease played no role in design, implementation, analysis, interpretation or decision to report these results. 
Conclusions-Total serum testosterone is associated with an increased risk of both advanced hepatic fibrosis and advanced hepatic inflammatory activity in HCV-infected men. Testosterone may be important in the pathogenesis of HCV-related advanced liver disease in males.

\section{MeSH Keywords}

epidemiology; digestive system; hormones; steroids; reproductive and urinary physiological phenomena; diagnosis

\section{Introduction}

It is estimated that $1.6 \%$ of the U.S population ( $>4.1$ million persons) is hepatitis $\mathrm{C}$ virus (HCV) infected. ${ }^{1}$ From $75-85 \%$ develop chronic infection, ${ }^{2}$ with an estimated $16 \%{ }^{3}-33 \%{ }^{4}$ of the chronically $\mathrm{HCV}$-infected subsequently developing cirrhosis within 20-25 years, and likely more with longer follow-up. Chronic HCV is now the leading cause of cirrhosisrelated liver transplant ${ }^{5}$ and $\mathrm{HCC}^{6}$ in the U.S.

Males have strikingly increased risk of advanced liver disease (cirrhosis and HCC) across varied disease etiologies including HCV; thus supporting the potential roles for genderassociated differences in risk factor exposures as well as sex-based biological differences in disease progression. Several studies performed in large population-based cohorts have examined the association between testosterone and risk of hepatitis B virus associated HCC in males, ${ }^{7-10}$ with three performed in a Taiwanese cohort finding increased serum testosterone was associated with increased risk of $\mathrm{HBV}$-associated HCC. ${ }^{7-9}$

However, only a few very small studies have examined the association between testosterone and advanced liver disease or HCC specifically in the background of HCV. ${ }^{11-13}$ A small prospective cohort in Japanese 46 males with HCV-related cirrhosis found that increased serum total testosterone was associated with increased HCV-related HCC risk. ${ }^{13}$ In contrast, a case-control study in $35 \mathrm{HCV}$-infected Australians found no difference in mean serum total testosterone level between an advanced fibrosis case group compared to mild fibrosis control group, ${ }^{11}$ though the study had inadequate power to adjust for factors known to be associated with both liver disease risk and testosterone levels including age and current alcohol use.

Examining the role of testosterone in HCV-related liver disease has the potential of explaining the male predominance in liver disease, expanding markers of advanced liver disease, and providing important clues to the pathogenesis of HCV-related liver disease. One population disproportionately affected by the HCV epidemic includes the over 8 million veterans of the U.S. armed services enrolled in the Department of Veteran Affairs (VA) healthcare system. ${ }^{14}$ A blinded national seroprevalence study found the HCV prevalence rate in these veterans was over 3-times higher than that reported for the overall U.S. population. ${ }^{15}$

We performed a cross-sectional study in a large multiethnic population of U.S. male veterans with chronic $\mathrm{HCV}$ to examine the association between total serum testosterone and risk of advanced hepatic fibrosis and inflammatory activity based on the validated serological FibroSURE-ActiTest. ${ }^{16}$ 


\section{Methods}

\section{Population and study design}

We performed a cross-sectional study at the Michael E. DeBakey VA Medical Center (MEDVAMC). We prospectively recruited all consecutive veterans eligible for study participation at their scheduled clinic appointments at the dedicated Hepatitis $\mathrm{C}$ primary care clinic between May 1, 2009 and November 1, 2010. VA healthcare policy mandates all veterans who receive either a diagnostic code for $\mathrm{HCV}$ or have a positive laboratory test are automatically scheduled for an appointment in this clinic for clinical evaluation and patient education.

\section{Recruitment and study eligibility}

The electronic medical records of all veterans with scheduled HCV clinic visits were screened by trained research assistants to determine study eligibility based on: 1) presence of at least one diagnostic code or laboratory value suggestive of $\mathrm{HCV}, 2$ ) aged 18 to 70 years, 4) no history of co-infection with either HIV or HBV, liver transplant, decompensated liver disease, hepatocelluar carcinoma (HCC), psychosis or dementia. We restricted our current analysis to veterans who met the following additional eligibility criteria: 1) male; 2) serologically confirmed presence of chronic infection (HCV viremia) without HIV or HBV co-infection; 3) not currently receiving HCV treatment; 4) no self-reported medical diagnoses, procedures, or medication usage that might substantially alter sex hormones, including a diagnosis of testicular cancer or treated prostate cancer, use of estrogen or androgen therapy (e.g., testosterone transdermal patch), or use of other gonadal hormone agonist/antagonists (e.g., flutamide), 5) had valid FibroSURE-ActiTest results; and 6) completed all primary study measures as of November 30, 2010.

This research was approved by the Institutional Review Boards for the Michael E. DeBakey VA Medical Center and Baylor College of Medicine.

\section{Data Collection and Study Measures}

Surveys-RAs administered a computer-assisted patient risk factor and medical history survey. We collected data on: potential mode and time intervals of likely exposures to HCV employing questions validated from a previous national survey of $\mathrm{HCV}$ in veterans using the VA; ${ }^{17,18}$ lifetime history and current use of alcohol use and smoking using questions created for a population-based GI cohort research; ${ }^{19}$ and lifetime medical history, including occurrence, timing, and medical management, of liver disease as well as cancers including prostate and testicular cancer. Survey responses were used to further estimate: 1) maximum duration of infection as the difference between self-reported age at first exposure to a known risk factor, with IDU and blood transfusion prior to 1992 given precedence, and date of study enrollment, and 2) chronic alcohol abuse defined as current or previous self-reported history of history of consuming at least 3 drinks a day for at least 10 consecutive years, with 1 drink defined as 12 ounces of beer, 4 ounces of wine, or 1.5 ounces of liquor.

Anthropometric measurements-RAs measured participant body weight in bare feet using the InBody520 Direct Segmental 8-point Multi-frequency Bioelectrical Impedance Analysis scale, which has $98 \%$ correlation with dual-energy X-ray absorptiometry (DXA) and $99 \%$ reproducibility. ${ }^{20,21}$ Height in inches was also obtained using a study designated stadiometer, and was entered directly into the scale for calculation of BMI using the Quetlet index formula weight in pounds $\times 703 /$ squared height in inches.

Serology-The CLIA-certified MEDVAMC Central Laboratory Service tested for HCV antibodies using immunometric immunoassay (Ortho Clinical Diagnostics), HCV genotype 
using the InnoLiPA HCV II (Siemens) and quantitative viral load using the COBAS TaqMan HCV Test (Roche). Total serum testosterone was measured using a competitive binding immunoenzymatic assay (Beckman Coulter). Diabetes mellitus was defined as present if fasting glucose was above $126 \mathrm{mg} / \mathrm{dl}$ or if non-fasting glucose was above $200 \mathrm{mg}$ / $\mathrm{dl}$, or if a glucose test was not performed because of previous medical record documented presence of diabetes. Laboratory personnel were blinded to survey and FibroSURE-ActiTest results.

FibroSURE-ActiTest-Hepatic disease severity was assessed in a CLIA-certified commercial laboratory using the FibrosSURE-ActiTest referred to in Europe as the FibroTEST-ActiTest (BioPredictive, France). The FibroSURE uses a proprietary algorithm utilizing a 2-macroglobulin, apolioprotein A1, haptoglobin, total bilirubin, and $\gamma$-glutamyltranspeptidase to determine fibrosis level and further adds alanine transaminase to determine necroinflammatory activity level. In addition to providing a total quantitative scale score, it further classifies those scores into METAVIR biopsy-based equivalent degree of hepatic fibrosis (F0, no fibrosis present - F4, cirrhosis) and inflammatory activity (A0, no inflammatory activity - A3, severe inflammatory activity). It has been demonstrated to have high concordance with biopsy-assessed level of METAVIR pathology and demonstrates strong test performance characteristics in diverse HCV-infected populations. ${ }^{16,22-27}$

Quality control—In addition to database encoded internal validity datachecks, we also performed quality control analyses for primary study measures via a random $1 \%$ doubleentry verification sub-study. Overall error rate was less than $0.5 \%$ with all discrepancies resolved.

Analysis-We classified all study participants by applying a priori study-established criteria to FibroSURE results as either advanced fibrosis cases (F4, F3/F4 and F3) or mild/ absent fibrosis controls (F0-F2/F3), and as advanced inflammation cases (A3, A2/A3, A2) or mild/no inflammation controls (A0-A1/A2). Baseline sociodemographic, risk factor history, and clinical variables were compared in advanced disease cases of hepatic fibrosis and hepatic inflammation compared to their respective mild disease controls using a $\chi^{2}$ and Fisher's exact test for large and small sample categorical variables, respectively, and independent sample T-test and the Brown-Mood median test for means of normally distributed variables and medians of non-normally distributed variables, respectively.

We calculated an estimated Fibrosis Progression Rate (FPR) by adapting the method of Poynard et al $(1997)^{4}$ to be the ratio between FibroSURE-determined METAVIR numeric fibrosis stage (0-4) divided by the estimated maximum duration of infection. It assumes a constant rate of fibrosis progression, that fibrosis was absent prior to HCV infection, and that the aggregate use of estimated first exposure as actual infection date is not systematically biased. We evaluated if the median FPR increased across increasing tertiles of total serum testosterone using the non-parametric Jonckheere-Terpstra test for ordered alternatives.

We employed unconditional binary logistic regression analysis to create two sets of multivariate models to evaluate the association between total serum testosterone and risk of advanced hepatic fibrosis and advanced hepatic inflammation, respectively. All models adjusted for potential confounding by factors known to be independently related to both risk of advanced liver disease and to serum testosterone levels/testosterone signaling including: age, chronic alcohol abuse, viral load, race/ethnicity, adiposity (BMI 225 ), and diabetes mellitus (DM). All models were assessed for goodness of fit with the Hosmer-Lemeshow test, with all effect estimates reported as odds ratios with associated $95 \%$ confidence intervals. All multivariate analyses were performed with SPSS 18 (Somers, NY). We 
calculated the non-parametric Spearman rank order correlation coefficient $(\rho)$ as another method to assess strength of association and potential multicollinearity between our main risk factor of interest, total serum testosterone, and confounders measured on a continuous scale including age and viral load. We also performed an exploratory evaluation for potential interaction between all first-level main effects, with interaction terms reported in the final models if statistically significant.

\section{Results}

We prospectively recruited 325 male veterans ( $80 \%$ consent rate) who met our initial screening eligibility criteria. We subsequently excluded 17 patients because of a nondetectable viral load $(n=3)$, positive HIV or HBV surface antigen $(n=4)$, or a history of testicular cancer, medically or surgically managed prostate cancer, or use of a sex hormonealtering pharmacotherapy $(\mathrm{n}=10)$.

Most (93\%) of our 308 chronically HCV infected male veteran study participants were between ages 45 and 64 years old and were of either African-American or White race/ ethnicity (52\% and $43 \%$, respectively). (Table 1) Comorbid risk factors were highly prevalent in this population, with $34 \%$ reporting a positive lifetime history of chronic alcohol abuse, $51 \%$ a positive history of injection drug use (IDU), and $71 \%$ study-classified as overweight or obese (BMI 25). (Table 1) A maximum estimated duration of HCV infection of 34 years (or estimated earliest median age at infection of 22 years old) was calculated based upon data provided by the $90 \%$ of respondents who reported both a positive history of, as well as correspondent ages for, at least one established risk factor for HCV transmission (e.g., blood transfusion pre-1992 or injection drug use). Less than 5\% of study participants had ever received anti-HCV therapy.

FibroSURE-determined METAVIR equivalent hepatic pathology was used to stratify our study sample into 105 cases with advanced fibrosis $\left(\mathrm{n}_{\mathrm{F} 4}=92\right.$ and $\left.\mathrm{n}_{\mathrm{F} 3 / \mathrm{F} 4}=13\right)$ and 203 mild fibrosis controls $\left(\mathrm{n}_{\mathrm{F} 0}=15, \mathrm{n}_{\mathrm{F} 0 / \mathrm{F} 1}=15, \mathrm{n}_{\mathrm{F} 1}=7, \mathrm{n}_{\mathrm{F} 1 / \mathrm{F} 2}=48, \mathrm{n}_{\mathrm{F} 2}=42, \mathrm{n}_{\mathrm{F} 3}=76\right)$; and into 88 cases with advanced inflammatory activity $\left(\mathrm{n}_{\mathrm{A} 3}=82\right.$ and $\left.\mathrm{n}_{\mathrm{A} 2 / \mathrm{A} 3}=6\right)$ and 220 mild inflammatory activity controls $\left(\mathrm{n}_{\mathrm{A} 0}=51, \mathrm{n}_{\mathrm{A} 0 / \mathrm{A} 1}=49, \mathrm{n}_{\mathrm{A} 1}=25, \mathrm{n}_{\mathrm{A} 1 / \mathrm{A} 2}=66\right.$, and $\left.\mathrm{n}_{\mathrm{A} 2}=29\right)$. Total serum testosterone generally increased with increased fibrosis and inflammatory activity (F0$\mathrm{F} 1=4.8, \mathrm{~F} 1-\mathrm{F} 2=4.5, \mathrm{~F} 2=5.6, \mathrm{~F} 3=5.4, \mathrm{~F} 3 / \mathrm{F} 4-\mathrm{F} 4=5.9 \mathrm{ng} / \mathrm{ml}$, and A0-A1=5.2, A1-A1/A2= 5.1, $\mathrm{A} 2=5.6, \mathrm{~A} 2 / 3-\mathrm{A} 3=5.7 \mathrm{ng} / \mathrm{ml}$, respectively). These trends were even more apparent when median testosterone was calculated in samples with a restricted age range (e.g., 50-54 yrs) in whom baseline total testosterone levels would be expected to be more similar. (data not shown)

Both advanced fibrosis cases as well as advanced inflammatory activity cases had higher testosterone levels compared to their mild disease controls $(6.0 \mathrm{ng} / \mathrm{ml} v \mathrm{vs} .5 .3 \mathrm{ng} / \mathrm{ml}$ and 5.9 $\mathrm{ng} / \mathrm{ml}$ vs. $5.4 \mathrm{ng} / \mathrm{ml}$, respectively), with this mean difference highly significant for advanced fibrosis ( $\mathrm{p}=0.009$ ). (Table 2 ) There was modest significant negative correlation between median age and total serum testosterone and between BMI and testosterone $\left(\rho_{\text {Spearman }}=\right.$ $-0.12, \mathrm{p}=0.04$, and $\rho_{\text {Spearman }}=-0.23, \mathrm{p}<0.001$, respectively), but no significant correlation between testosterone and HCV viral load ( $\rho_{\text {Spearman }}=0.03, p=0.56$ ).

Diabetes mellitus was also significantly more prevalent in both advanced hepatic fibrosis cases and advanced hepatic inflammatory activity cases $(\mathrm{p}<0.001$ and $\mathrm{p}=0.003$, respectively). Although mean age was also significantly higher in those with advanced fibrosis compared to mild fibrosis controls ( $\mathrm{p}=0.02$ ), mean age was not similarly higher in advanced inflammation cases. Baseline distribution of all other potential confounders evaluated, including a history of chronic alcohol abuse, viral load, overweight/obesity and 
ethnicity, were not significantly different among advanced hepatic disease cases and their respective mild disease controls. (Table 2)

Higher serum testosterone levels were associated with increased relative risk of advanced FibroSURE-determined hepatic fibrosis (F3/F4 and F4) in both univariate and multivariate analyses. (Table 2) Further, the adjusted 3.78-fold and 2.18-fold excess fibrosis risk in HCV + male veterans with medium and upper testosterone levels, respectively, in comparison to those with lower testosterone levels suggests a monotonic positive dose-response relationship. There was no evidence of significant interaction between any main effects. The inclusion of all other known or biologically-plausible confounders like diabetes and adiposity evaluated in the multivariate model further enhanced both the magnitude and apparent dose-response relationship of the observed association between higher total testosterone levels and increased advanced hepatic fibrosis risk. Overall, we observed a significant $27 \%$ increase in advanced hepatic fibrosis risk for each $1 \mathrm{ng} / \mathrm{ml}$ increase in total serum testosterone $\left(\mathrm{OR}_{\text {adjusted }}=1.27,95 \%\right.$ CI 1.13-1.44, $\left.\mathrm{p}<0.001\right)$. (data not shown) Median fibrosis progression rates (FPR) also significantly increased across tertiles of total serum testosterone (FPRs=0.067, 0.079, and 0.095 for the lower, middle, and upper tertiles, respectively, $\mathrm{p}<0.001)$.

Higher serum testosterone levels were significantly associated with risk of advanced FibroSURE-determined hepatic inflammatory activity (A2/A3 and A3) in both univariate and multivariate analyses (range 1.9-2.7- fold significant excess relative risk). (Table 3) Also similar were our findings that the magnitude of the association between increased testosterone levels and increased inflammatory disease risk was further modestly strengthened in multivariate in comparison to univariate analysis (e.g.,

$\mathrm{OR}_{\text {univariate highest testosterone level }}=1.92,95 \% \mathrm{CI}$ vs. $\mathrm{OR}_{\text {multivariate highest testosterone level }}=2.64$, $95 \% \mathrm{CI}$, respectively), and that there was no evidence of significant interaction among any main effects. However, in contrast to our findings for advanced fibrosis, there was no apparent dose-response relationship between total serum testosterone level and advanced inflammatory activity risk. (Table 3 ) Overall, we found that a $1 \mathrm{ng} / \mathrm{ml}$ increase in total serum testosterone was associated with a significant $16 \%$ increase in advanced inflammatory activity risk $\left(\mathrm{OR}_{\text {adjusted }}=1.16,95 \% \mathrm{CI} 1.02-1.31, \mathrm{p}=0.02\right)$. (data not shown)

The Hosmer-Lemeshow test results were supportive of fit for all reported models. We also performed several sensitivity analyses to evaluate the robustness of our observed associations of excess advanced hepatic disease risk with increased total serum testosterone. In our first set of sensitivity analyses, we restricted our analysis to the $84 \%$ of our HCV+ male veteran sample with total testosterone within a normal range for adult males (2.41-8.47 $\mathrm{ng} / \mathrm{ml}$ ). We continued to observe a significant dose-response between higher total serum testosterone levels and advanced hepatic fibrosis $\left(\mathrm{OR}_{\text {adjusted }}=3.90,95 \% \mathrm{CI} 1.82-8.38\right.$ and $\mathrm{OR}_{\text {adjusted }}=2.25,95 \% \mathrm{CI} 1.05-4.80$ for advanced fibrosis risk for those in the upper and middle testosterone level as compared to those in the lower testosterone level, respectively). (Table 4) We similarly found that increased testosterone level also continued to be associated with advanced inflammatory activity risk $\left(\mathrm{OR}_{\text {adjusted }}=2.66,95 \% \mathrm{CI} 1.19-5.99\right.$ and $\mathrm{OR}_{\text {adjusted }}=3.12,95 \%$ CI $1.41-6.92$ for advanced inflammatory activity risk for those in the upper and middle testosterone level as compared to those in the lower testosterone level, respectively). In a second set of sensitivity analyses where we changed the advanced disease case definitions to be more and less restrictive respectively, we continued to demonstrate substantial excess risk of both advanced hepatic fibrosis and advanced hepatic inflammatory activity with increased total serum testosterone levels. (Table 4) 


\section{Discussion}

Our study demonstrated that higher total serum testosterone levels are associated with an increased risk of advanced FibroSURE-ActiTest (FibroSURE)-determined hepatic fibrosis and inflammatory activity in chronically hepatitis $\mathrm{C}$ virus infected (HCV+) male veterans. The magnitude of the observed excess risk of both advanced fibrosis and advanced inflammatory activity was independent of other known or biologically-plausible strong potential confounders including diabetes mellitus, age, viral load, race/ethnicity, adiposity, and chronic alcohol abuse. Our findings of excess testosterone-associated risk persisted in multiple sensitivity analyses.

Few studies have specifically examined the association between testosterone and degree of HCV-related liver disease. Two smaller case-control studies performed in Australian $(\mathrm{N}=35)^{11}$ and Iranian $(\mathrm{N}=82)^{12} \mathrm{HCV}$-infected males reported discordant results for total testosterone; one study found no difference in mean total testosterone levels in cases with advanced fibrosis compared to mild fibrosis controls, ${ }^{11}$ while the other found substantially reduced mean total testosterone levels in advanced fibrosis cases. ${ }^{12}$ Both reported that free testosterone was significantly lower among the advanced fibrosis case group compared to their mild fibrosis control group. However, likely due to sample size restriction, neither performed multivariate analyses to evaluate how these group mean differences may be related to confounding due to differential distribution of other risk factors like diabetes mellitus. Other potential explanations for the discordant findings of our study and those studies include differences in underlying target populations, including the Iranian study's restriction to males without a history of alcohol abuse (drink $>20 \mathrm{~g} /$ day), ${ }^{12}$ and our use of FibroSURE-determined hepatic pathology. Similar to our overall findings, a third prospective cohort study performed in Japanese males with HCV-related cirrhosis ( $\mathrm{N}=46)$ found a significant adjusted 9.1-fold excess relative risk of hepatocellular carcinoma among men with total testosterone in the upper tertile. ${ }^{13}$ Overall, our consistent findings of substantial excess total testosterone-associated risk in a large, multiethnic sample suggests the importance of additional studies to further assess its possible role in pathogenesis and as a risk marker for advanced fibrosis and inflammation in chronically $\mathrm{HCV}$-infected males.

Our findings of excess testosterone-associated risk in $\mathrm{HCV}+$ males are consistent with findings of increased liver cancer risk in males who abuse anabolic steroids, ${ }^{28}$ in women with hyperandrogenemia secondary to polycystic ovarian disease, ${ }^{29}$ in androgen-treated Fancomia anemia patients, ${ }^{28}$ and in HBV+ Taiwanese males with higher serum testosterone levels. ${ }^{7-9}$ Several experimental studies have demonstrated that the HBV virus augments androgen receptor (AR) signaling, ${ }^{30-32}$ with ligand-dependent AR activation associated with increased HBV RNA transcription in both animal and HepG2 cell-line models. ${ }^{33,34}$ A recent experimental study demonstrated the HCV virus also augments AR signaling. ${ }^{35}$ The AR, whose predominant ligand is testosterone, is a nuclear hormone receptor expressed in liver. In addition to regulating genes involved in sexual dimorphism, the AR regulates/coregulates numerous other genes involved in diverse cellular processes of relevance to liver disease including cellular proliferation, differentiation, apoptosis, and inflammatory response (e.g., EGFR, PTEN, TP53, and IL6ST).

Unfortunately, we do not have analogous direct measures of hepatic AR signaling, of additional sex hormones including other androgens or estrogens, or of metabolic byproducts of hepatic clearance of circulating androgens. Therefore, we are unable to determine if the observed excess risk of advanced liver disease with higher testosterone levels is an effect of increased hepatic androgen levels, increased expression of androgenresponsive genes, or decreased hepatic clearance of circulating androgens with advanced HCV-related disease. Although the liver is the primary site of metabolic clearance of 
circulating androgens, the vast majority of androgens are not metabolically cleared from circulation by the liver, but are rather metabolized locally in target tissues. This in conjunction with our finding that circulating total testosterone levels are higher with increased fibrosis prior to development of cirrhosis suggests that our observed associations are unlikely to be solely attributable to decreased hepatic clearance of circulating androgens. Additional research that includes direct measurement of androgen receptor gene expression and of other sex hormones will be useful in further clarifying the role testosterone plays in the etiopathogenesis of HCV-related disease.

Our study has multiple strengths including it is the largest study to examine the association between testosterone and HCV-related hepatic fibrosis risk to date as well as the first study to evaluate the association between testosterone and risk of advanced inflammatory activity. It was conducted in an ethnically diverse North American population. We employed a rigorous study design that included direct prospective recruitment of all study eligible male veterans with clinically scheduled visits at a VA Hepatitis $\mathrm{C}$ clinic. Together with our high participation rates, this helps mitigate concerns about potential selection bias. We also employed direct laboratory-based measures for our main outcome, FibroSURE-determined hepatic pathology, our main exposure, serologically-determined total serum testosterone, and for several other key confounders including glucose used for diabetes determination and viral load. Notably $>90 \%$ of participants completed the blood draw used to measure total testosterone in the morning helping to minimize potential impact of diurnal pulsatile fluctuation in testosterone levels. All laboratory tests were performed in a blinded and uniform manner by single CLIA-certified laboratories in order to decrease potential for both testing variability and tester/information bias. Additionally, both our study participants and research assistants were unaware of the participant's FibroSURE or testosterone test results at the time the survey was administered helping to minimize potential for recall or interviewer bias. Further, we obtained a detailed history of lifetime alcohol use necessary to adjust for chronic alcohol abuse, and also obtained and adjusted for direct clinical measures of adiposity, viral load and glucose-determined diabetes as, in addition to their well-known association with liver disease risk, all have been associated with significant alterations in androgen levels or androgen signaling. ${ }^{35-38}$ Finally, we performed multiple sensitivity analyses that suggested our findings of excess testosterone-associated liver disease risk in $\mathrm{HCV}+$ male veterans are robust.

However, our study also has limitations. The cross-sectional design prevents the temporal inference necessary to establish a casual role for testosterone in HCV-related liver disease. Additionally, although the validated FibroSURE has been shown in multiple studies in $\mathrm{HCV}$-infected populations to have good test performance characteristics, ${ }^{16,22-25}$ hepatic biopsy is still considered the gold-standard to diagnose individual patients. However, biopsies are known to be associated with variability in both sampling and pathologist evaluation, and they are invasive and costly, thus limiting their use in large epidemiological studies such as ours. Future research will be needed to determine if this total testosterone associated risk of advanced FibroSURE-determined hepatic pathology in our HCV+ male veterans is replicated among those with hepatic biopsy. We also have only a single measure of the primary male androgen, total testosterone. It would have been desirable to have multiple measurements of total testosterone to mitigate intra-individual sampling and laboratory testing variability. However, we expect that any variability in sampling or testing would be non-differential between advanced disease cases and mild disease controls, and therefore any associated bias would be toward the null. It would also have been desirable to have directly measured or calculated free and weakly bound testosterone. However, total testosterone correlates well with free testosterone in aging males, ${ }^{39}$ has been shown to be reliably associated with increased risk of diabetes ${ }^{38}$ and has been shown to be a more valid predictor of androgen deficiency function than either directly measured free testosterone or 
calculated bioavailable testosterone. ${ }^{39}$ Total testosterone is also much more widelyaccessible, and more reliably measured than is directly measured free testosterone. ${ }^{40}$ Finally, due to sample size constraints, we were able to perform only an exploratory evaluation of interaction between total testosterone and other risk factors of interest including age and adiposity. Although we found no significant interactions, additional larger studies will be needed to determine if there are subgroups at particularly increased risk of HCV-related liver disease progression in the background of higher total testosterone levels.

In conclusion, we demonstrated that total serum testosterone is associated with substantially increased risk of both advanced hepatic fibrosis and advanced hepatic inflammatory activity in chronically HCV-infected male veterans accessing the VA healthcare system. The strength and consistency of this excess testosterone-associated liver disease risk suggests total serum testosterone may play a role in disease progression in $\mathrm{HCV}+$ males. Future research will be important in assessing if increased total testosterone levels similarly increase relative risk of advanced HCV-related liver disease in $\mathrm{HCV}+$ women, and in determining if androgens including testosterone have potential therapeutic implications in $\mathrm{HCV}+$ men.

\section{Acknowledgments}

This material is based upon work supported in part by a VA Clinical Research and Development Merit Review Award (H-22934, PI: H. El-Serag, MD, MPH), and the National Institute of Diabetes Digestive and Kidney Diseases (NIDDK) [DK081736-01 and DK078154-03, PIs, D. White and H. El-Serag respectively] and the Houston VA HSR\&D Center of Excellence [HFP90-020]. We thank Dallese Jones, MA, and Jodi Francis, RN for their assistance with data collection, and Susanne Denk, BA and Adrian Rideau, BA for their research administrative assistance.

\section{Abbreviations}

$\begin{array}{ll}\text { HCV } & \text { hepatitis C virus } \\ \text { HBV } & \text { hepatitis B virus } \\ \text { HCC } & \text { hepatocellular carcinoma } \\ \text { OR } & \text { odds ratio MeSH }\end{array}$

\section{Reference List}

1. Armstrong GL, Wasley A, Simard EP, McQuillan GM, Kuhnert WL, Alter MJ. The prevalence of hepatitis C virus infection in the United States, 1999 through 2002. Ann Intern Med. 2006; 144:705-714. [PubMed: 16702586]

2. CDC (Centers for Disease Control and Prevention). The ABCs of hepatitis. 2009; 2009 http:// www.cdc.gov/hepatitis/Resources/Professionals/PDFs/ABCTable.pdf.

3. Thein HH, Yi Q, Dore GJ, Krahn MD. Estimation of stage-specific fibrosis progression rates in chronic hepatitis $C$ virus infection: a meta-analysis and meta-regression. Hepatology. 2008; 48:418431. [PubMed: 18563841]

4. Poynard T, Bedossa P, Opolon P. Natural history of liver fibrosis progression in patients with chronic hepatitis C. The OBSVIRC, METAVIR, CLINIVIR, and DOSVIRC groups. Lancet. 1997; 349:825-832. [PubMed: 9121257]

5. Wiesner RH, Sorrell M, Villamil F. Report of the first International Liver Transplantation Society expert panel consensus conference on liver transplantation and hepatitis C. Liver Transpl. 2003; 9:S1-S9. [PubMed: 14586888]

6. Fattovich G, Stroffolini T, Zagni I, Donato F. Hepatocellular carcinoma in cirrhosis: incidence and risk factors. Gastroenterology. 2004; 127:S35-S50. [PubMed: 15508101] 
7. Yu MW, Cheng SW, Lin MW, et al. Androgen-receptor gene CAG repeats, plasma testosterone levels, and risk of hepatitis B-related hepatocellular carcinoma. J Natl Cancer Inst. 2000; 92:20232028. [PubMed: 11121465]

8. Yu MW, Yang YC, Yang SY, et al. Hormonal markers and hepatitis B virus-related hepatocellular carcinoma risk: a nested case-control study among men. J Natl Cancer Inst. 2001; 93:1644-1651. [PubMed: 11698569]

9. Yu MW, Chen CJ. Elevated serum testosterone levels and risk of hepatocellular carcinoma. Cancer Res. 1993; 53:790-794. [PubMed: 8381328]

10. Yuan JM, Ross RK, Stanczyk FZ, et al. A cohort study of serum testosterone and hepatocellular carcinoma in Shanghai, China. Int J Cancer. 1995; 63:491-493. [PubMed: 7591255]

11. Nguyen HV, Mollison LC, Taylor TW, Chubb SA, Yeap BB. Chronic hepatitis C infection and sex hormone levels: effect of disease severity and recombinant interferon-alpha therapy. Intern Med J. 2006; 36:362-366. [PubMed: 16732861]

12. Safarinejad MR, Kolahi AA, Iravani S. Evaluation of semen variables, sperm chromosomal abnormalities and reproductive endocrine profile in patients with chronic hepatitis C. BJU Int. 2010; 105:79-86. [PubMed: 19594736]

13. Tanaka K, Sakai H, Hashizume M, Hirohata T. Serum testosterone:estradiol ratio and the development of hepatocellular carcinoma among male cirrhotic patients. Cancer Res. 2000; 60:5106-5110. [PubMed: 11016636]

14. Department of Veterans Affairs. Office of Policy and Planning. Analysis of Unique Veterans Utilization of VA Benefits \& Services. Apr 29.2009 2009 http://www.va.gov/VETDATA/docs/ SpecialReports/uniqueveteransMay.pdf.

15. Dominitz JA, Boyko EJ, Koepsell TD, et al. Elevated prevalence of hepatitis C infection in users of United States veterans medical centers. Hepatology. 2005; 41:88-96. [PubMed: 15619249]

16. Halfon P, Bourliere M, Deydier R, et al. Independent prospective multicenter validation of biochemical markers (fibrotest-actitest) for the prediction of liver fibrosis and activity in patients with chronic hepatitis C: the fibropaca study. Am J Gastroenterol. 2006; 101:547-555. [PubMed: 16542291]

17. Mendenhall CL, Moritz T, Rouster S, et al. Epidemiology of hepatitis C among veterans with alcoholic liver disease. The VA Cooperative Study Group 275. Am J Gastroenterol. 1993; 88:1022-1026. [PubMed: 8317400]

18. Bini EJ, Brau N, Currie $S$, et al. Prospective multicenter study of eligibility for antiviral therapy among 4,084 U.S. veterans with chronic hepatitis C virus infection. Am J Gastroenterol. 2005; 100:1772-1779. [PubMed: 16086714]

19. Satia-Abouta J, Galanko JA, Potter JD, et al. Associations of total energy and macronutrients with colon cancer risk in African Americans and Whites: results from the North Carolina colon cancer study. Am J Epidemiol. 2003; 158:951-962. [PubMed: 14607803]

20. Malavolti M, Mussi C, Poli M, et al. Cross-calibration of eight-polar bioelectrical impedance analysis versus dual-energy X-ray absorptiometry for the assessment of total and appendicular body composition in healthy subjects aged 21-82 years. Ann Hum Biol. 2003; 30:380-391. [PubMed: 12881138]

21. Medici G, Mussi C, Fantuzzi AL, Malavolti M, Albertazzi A, Bedogni G. Accuracy of eight-polar bioelectrical impedance analysis for the assessment of total and appendicular body composition in peritoneal dialysis patients. Eur J Clin Nutr. 2005; 59:932-937. [PubMed: 15928682]

22. Imbert-Bismut F, Ratziu V, Pieroni L, et al. Biochemical markers of liver fibrosis in patients with hepatitis C virus infection: a prospective study. Lancet. 2001; 357:1069-1075. [PubMed: 11297957]

23. Patel K, Benhamou Y, Yoshida EM, et al. An independent and prospective comparison of two commercial fibrosis marker panels (HCV FibroSURE and FIBROSpect II) during albinterferon alfa-2b combination therapy for chronic hepatitis C. J Viral Hepat. 2009; 16:178-186. [PubMed: 19175870]

24. Grigorescu M, Rusu M, Neculoiu D, et al. The FibroTest value in discriminating between insignificant and significant fibrosis in chronic hepatitis $\mathrm{C}$ patients. The Romanian experience. $\mathrm{J}$ Gastrointestin Liver Dis. 2007; 16:31-37. [PubMed: 17410286] 
25. Poynard T, Imbert-Bismut F, Ratziu V, et al. Biochemical markers of liver fibrosis in patients infected by hepatitis $C$ virus: longitudinal validation in a randomized trial. J Viral Hepat. 2002; 9:128-133. [PubMed: 11876795]

26. Poynard T, Munteanu M, Ngo Y, et al. ActiTest accuracy for the assessment of histological activity grades in patients with chronic hepatitis $\mathrm{C}$, an overview using Obuchowski measure. Gastroenterol Clin Biol. 2010; 34:388-396. [PubMed: 20580175]

27. Morali G, Maor Y, Klar R, et al. Fibrotest-Actitest: the biochemical marker of liver fibrosis--the Israeli experience. Isr Med Assoc J. 2007; 9:588-591. [PubMed: 17877064]

28. Velazquez I, Alter BP. Androgens and liver tumors: Fanconi's anemia and non-Fanconi's conditions. Am J Hematol. 2004; 77:257-267. [PubMed: 15495253]

29. Vassilatou E, Lafoyianni S, Vryonidou A, et al. Increased androgen bioavailability is associated with non-alcoholic fatty liver disease in women with polycystic ovary syndrome. Hum Reprod. 2010; 25:212-220. [PubMed: 19887498]

30. Chiu CM, Yeh SH, Chen PJ, et al. Hepatitis B virus X protein enhances androgen receptorresponsive gene expression depending on androgen level. Proc Natl Acad Sci U S A. 2007; 104:2571-2578. [PubMed: 17259306]

31. Yang WJ, Chang CJ, Yeh SH, et al. Hepatitis B virus X protein enhances the transcriptional activity of the androgen receptor through c-Src and glycogen synthase kinase-3beta kinase pathways. Hepatology. 2009; 49:1515-1524. [PubMed: 19205031]

32. Zheng Y, Chen WL, Ma WL, Chang C, Ou JH. Enhancement of gene transactivation activity of androgen receptor by hepatitis B virus X protein. Virology. 2007; 363:454-461. [PubMed: 17335866]

33. Wang SH, Yeh SH, Lin WH, Wang HY, Chen DS, Chen PJ. Identification of androgen response elements in the enhancer I of hepatitis B virus: a mechanism for sex disparity in chronic hepatitis B. Hepatology. 2009; 50:1392-1402. [PubMed: 19670412]

34. Wu MH, Ma WL, Hsu CL, et al. Androgen receptor promotes hepatitis B virus-induced hepatocarcinogenesis through modulation of hepatitis B virus RNA transcription. Sci Transl Med. 2010; 2:32ra35.

35. Kanda T, Steele R, Ray R, Ray RB. Hepatitis C virus core protein augments androgen receptormediated signaling. J Virol. 2008; 82:11066-11072. [PubMed: 18768969]

36. Iturriaga H, Lioi X, Valladares L. Sex hormone-binding globulin in non-cirrhotic alcoholic patients during early withdrawal and after longer abstinence. Alcohol Alcohol. 1999; 34:903-909. [PubMed: 10659727]

37. MacDonald AA, Herbison GP, Showell M, Farquhar CM. The impact of body mass index on semen parameters and reproductive hormones in human males: a systematic review with metaanalysis. Hum Reprod Update. 2010; 16:293-311. [PubMed: 19889752]

38. Ding EL, Song Y, Malik VS, Liu S. Sex differences of endogenous sex hormones and risk of type 2 diabetes: a systematic review and meta-analysis. JAMA. 2006; 295:1288-1299. [PubMed: 16537739]

39. Christ-Crain M, Meier C, Huber P, Zimmerli L, Trummler M, Muller B. Comparison of different methods for the measurement of serum testosterone in the aging male. Swiss Med Wkly. 2004; 134:193-197. [PubMed: 15106032]

40. Diver MJ. Laboratory measurement of testosterone. Front Horm Res. 2009; 37:21-31. [PubMed: 19011286] 


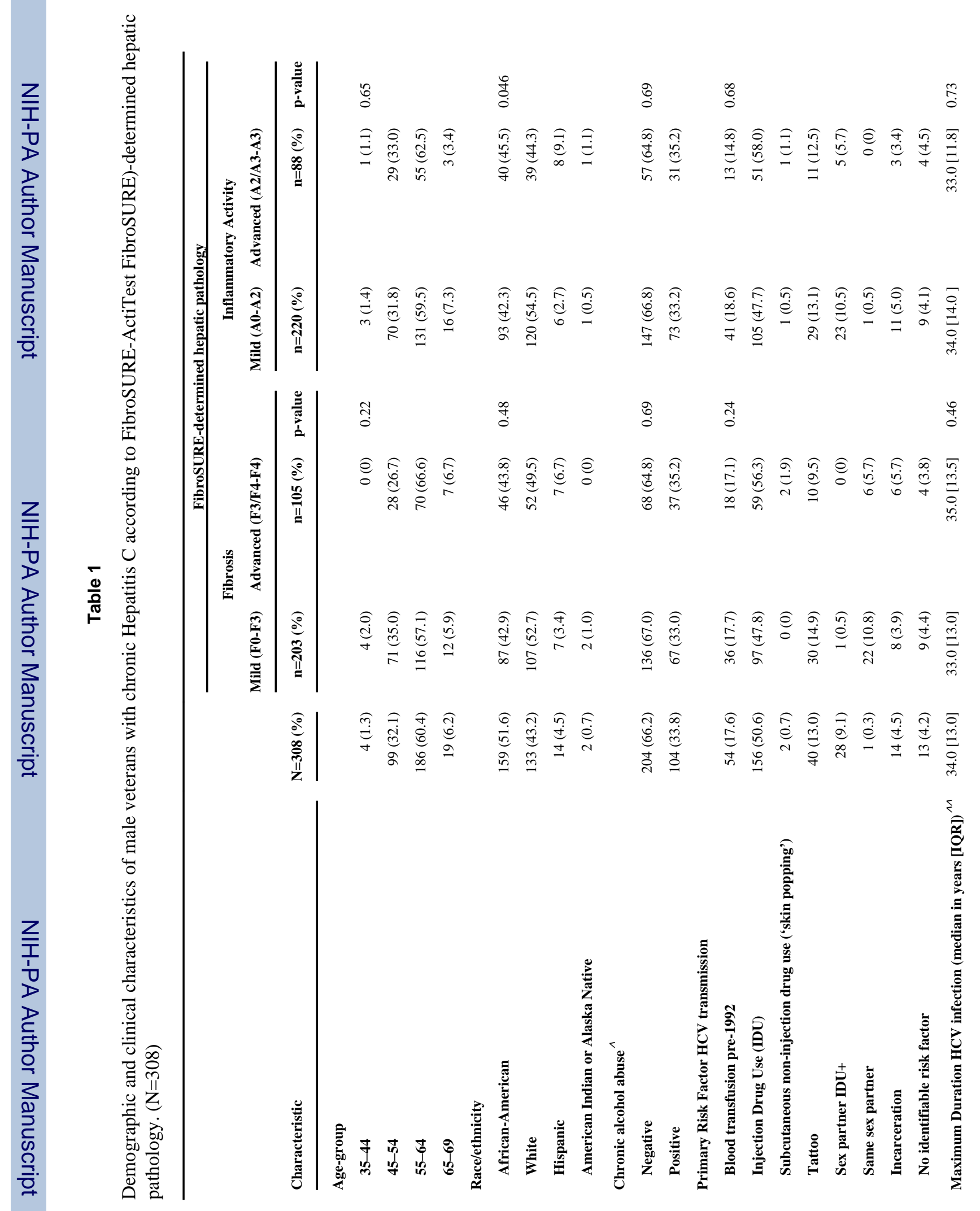




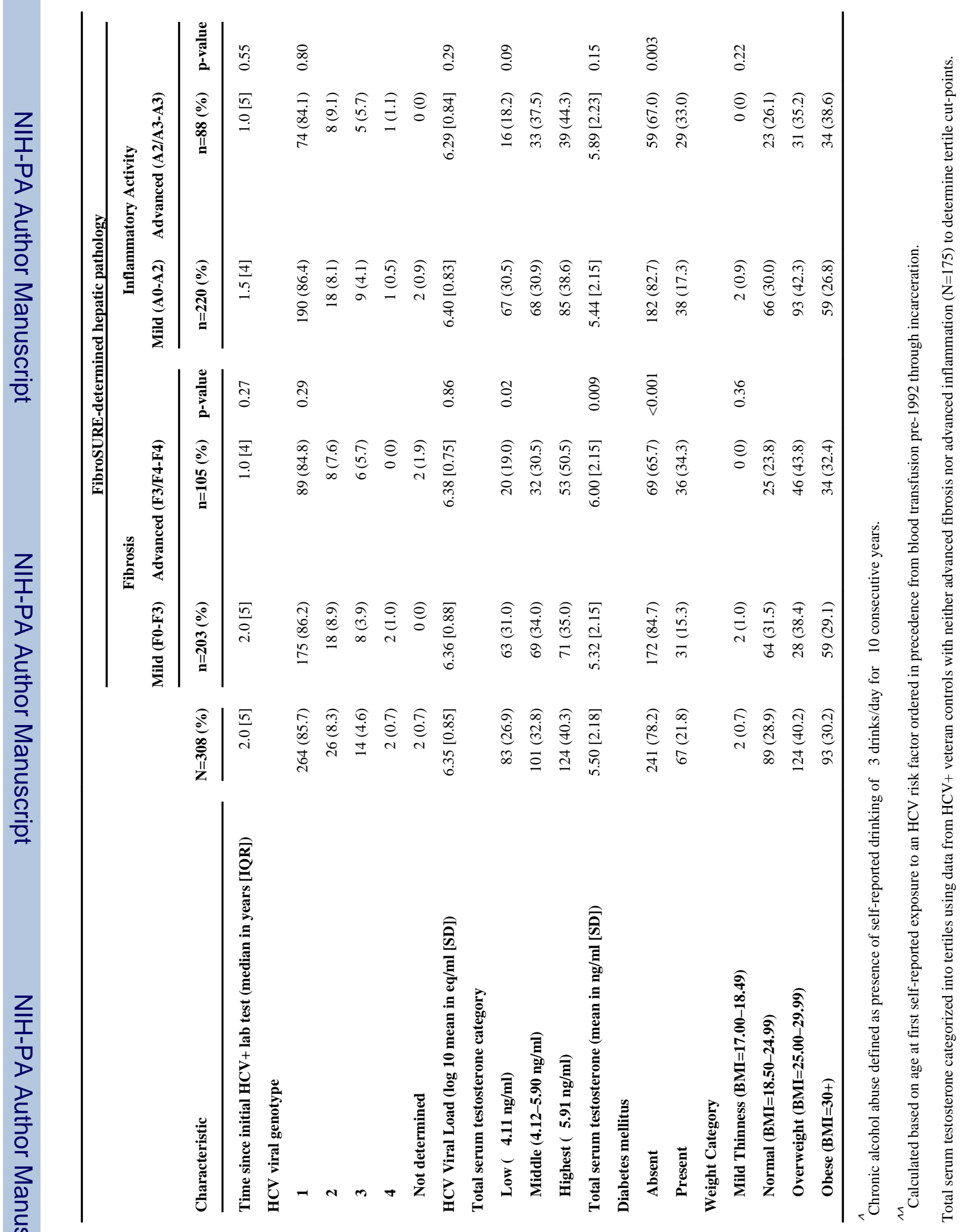

Hepatology. Author manuscript; available in PMC 2013 March 01. 


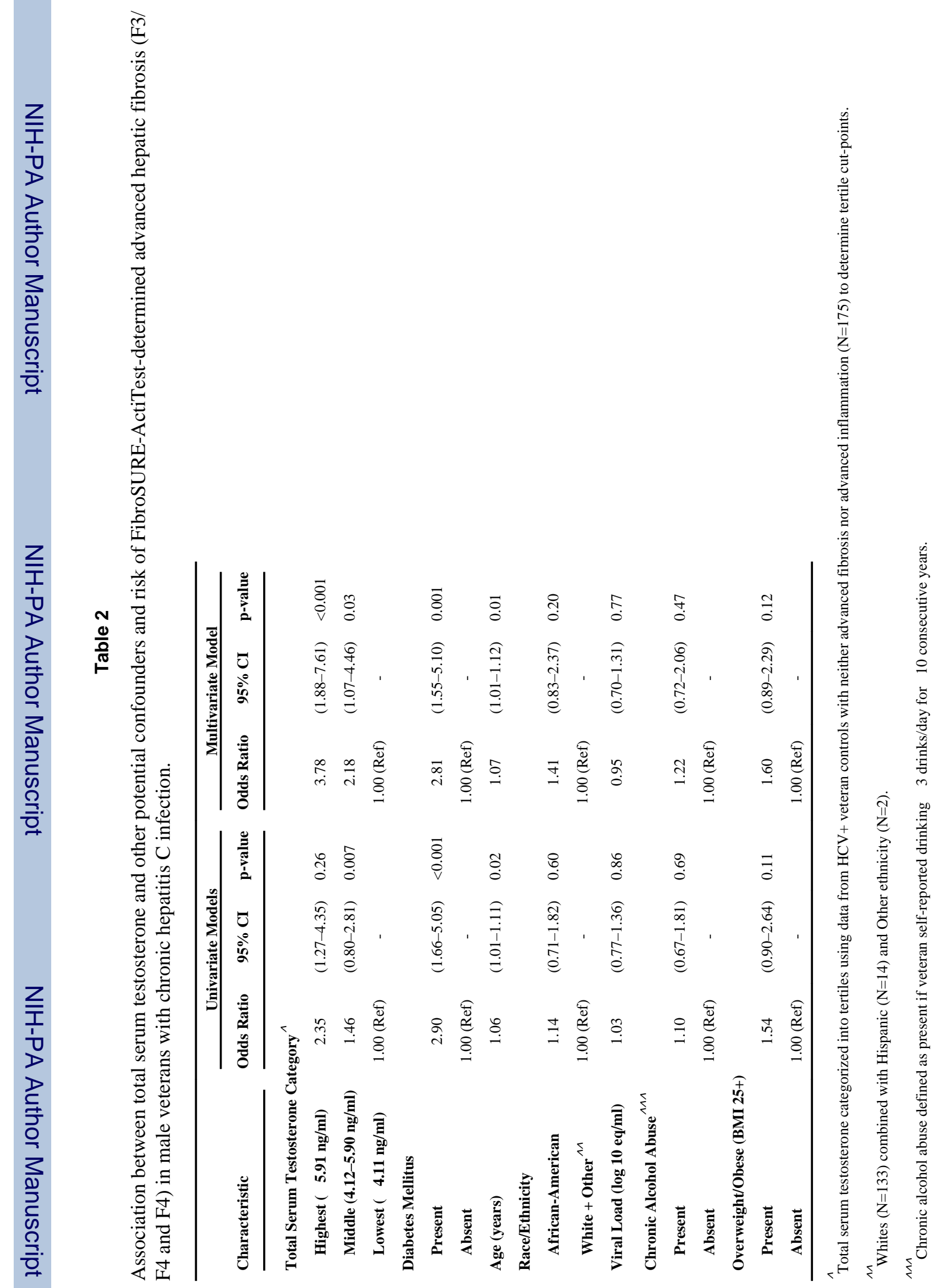

Hepatology. Author manuscript; available in PMC 2013 March 01. 


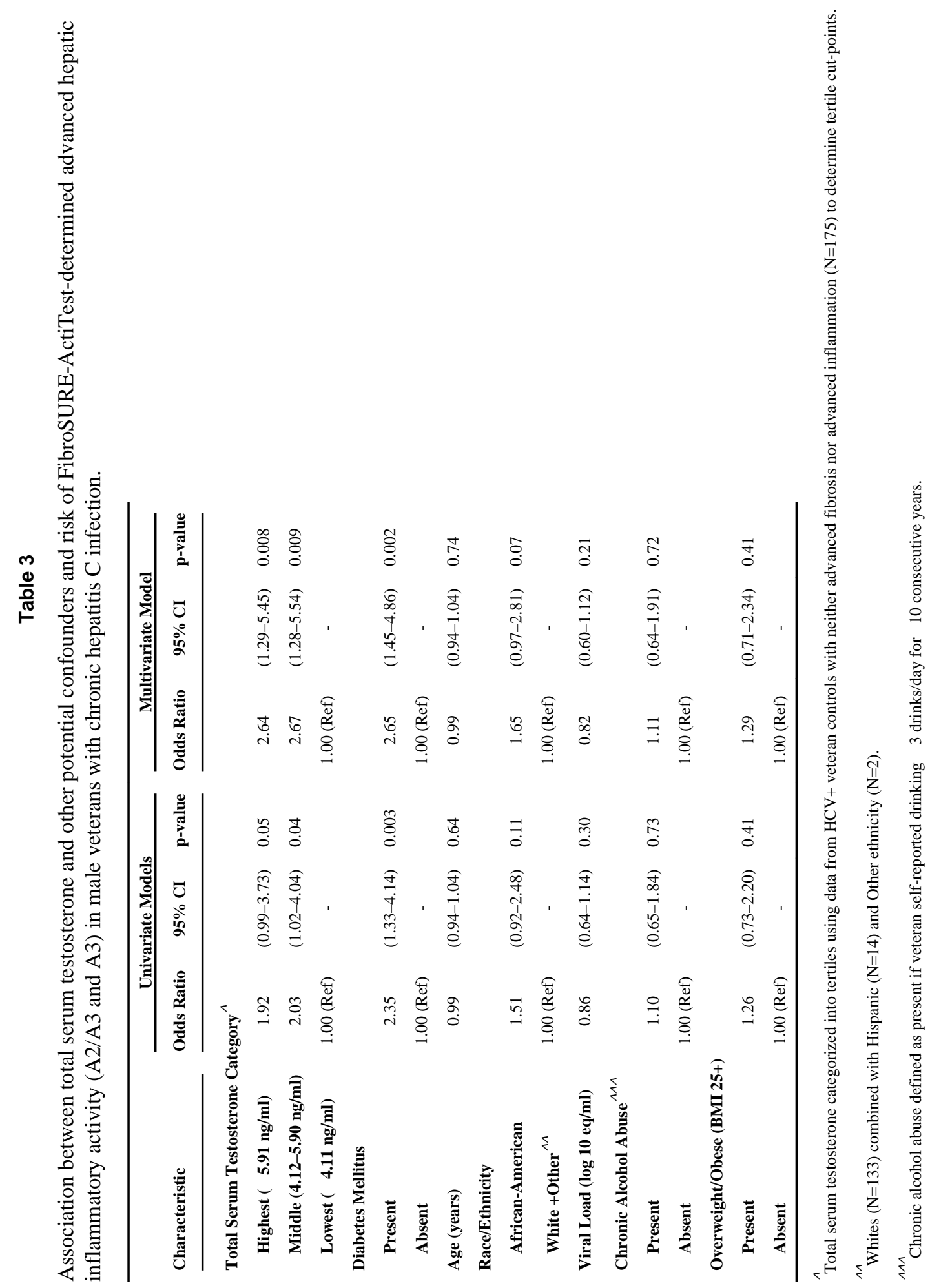

Hepatology. Author manuscript; available in PMC 2013 March 01. 


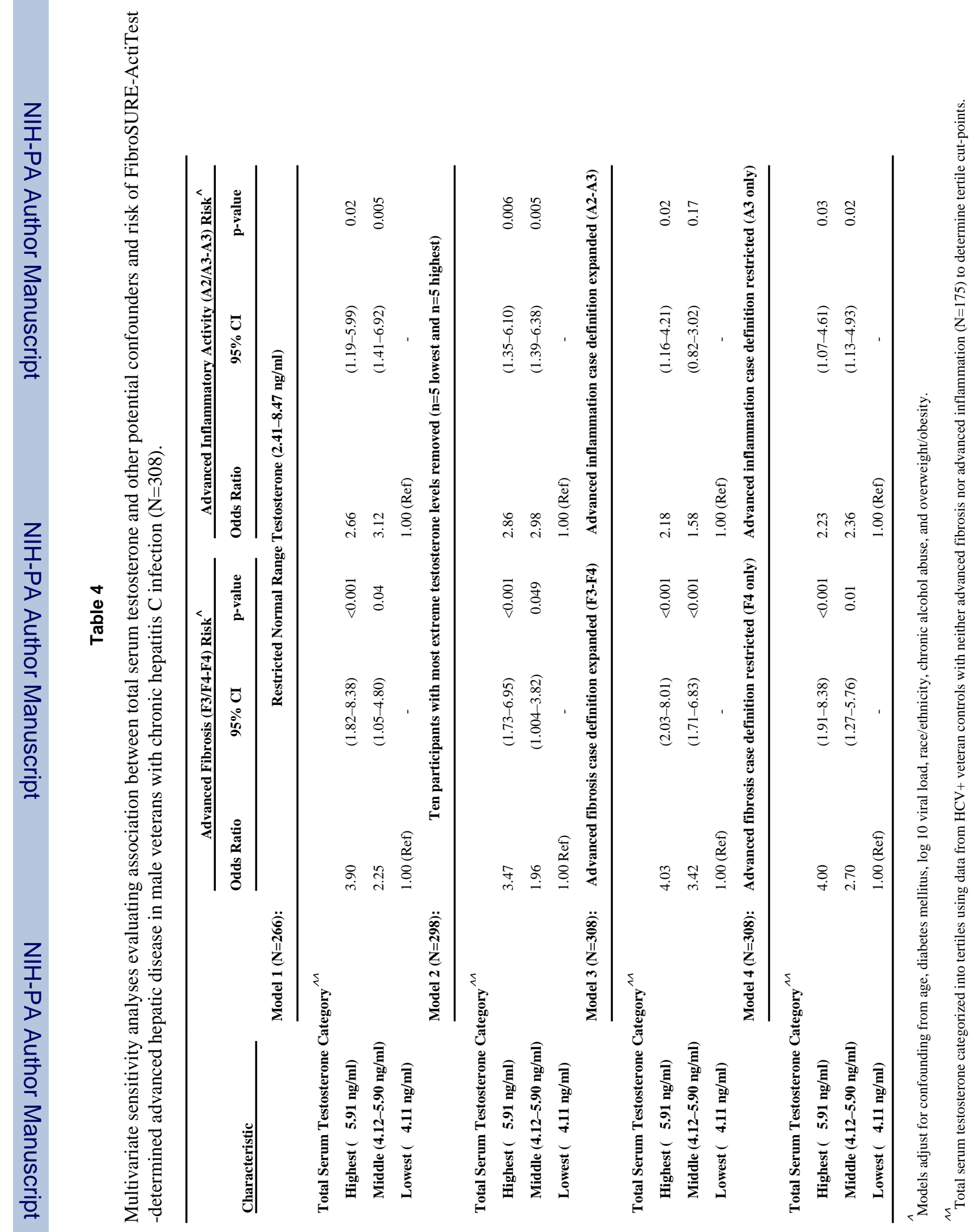

